Document downloaded from:

http://hdl.handle.net/10251/140905

This paper must be cited as:

Sanz Diaz, R.; García Gil, PJ.; Albertos Pérez, P.; Zhong, Q. (10-0). Robust controller design for input-delayed systems using predictive feedback and an uncertainty estimator. International Journal of Robust and Nonlinear Control. 27(10):1826-1840. https://doi.org/10.1002/rnc.3639

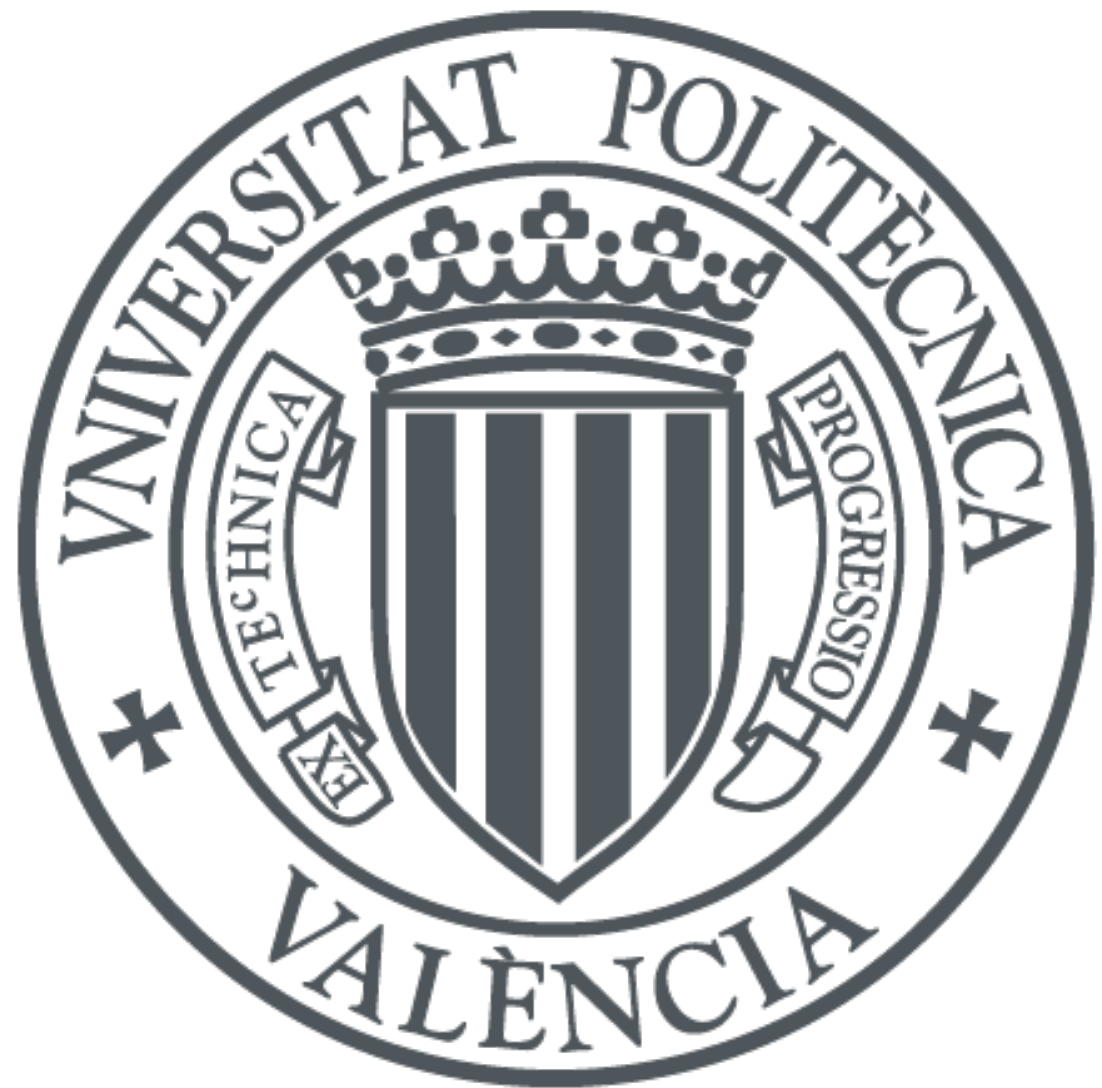

The final publication is available at

https://doi.org/10.1002/rnc.3639

Copyright John Wiley \& Sons

Additional Information 


\title{
Robust Controller Design for Input-Delayed Systems using Predictive Feedback and an Uncertainty Estimator
}

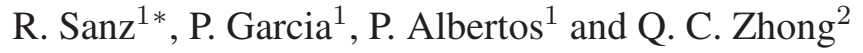 \\ ${ }^{1}$ Instituto de Automática e Informática Industrial, Universitat Politècnica de València, 46022 Spain \\ ${ }^{2}$ Dept. of Electrical and Computer Engineering, Illinois Institute of Technology. Chicago, IL 60616, USA
}

\begin{abstract}
SUMMARY
This paper deals with the problem of stabilizing a class of input-delayed systems with (possibly) nonlinear uncertainties by using explicit delay compensation. It is well known that plain predictive schemes lack robustness with respect to uncertain model parameters. In this work, an uncertainty estimator is derived for input-delay systems and combined with a modified state predictor, which uses current available information of the estimated uncertainties. Furthermore, based on Lyapunov-Krasovskii functionals, a computable criterion to check robust stability of the closed-loop is developed and cast into a minimization problem constrained to a linear matrix inequality (LMI). Additionally, for a given input delay, an Iterative-LMI algorithm is proposed to design stabilizing tuning parameters. The main results are illustrated and validated using a numerical example with a second-order dynamic system. Copyright (c) 2010 John Wiley \& Sons, Ltd.

Received ...
\end{abstract}

KEY WORDS: robust stabilization; input delay; state predictor; uncertainty estimator; LMI

\section{INTRODUCTION}

This paper deals with the problem of stabilizing a class of input-delayed systems with possibly nonlinear uncertainties. The stability analysis and control of time-delay systems is a problem of recurring interest because time delays are inherent to many engineering problems such as networked control systems, chemical processes, population dynamics or epidemic models [1]. Very often, time delays lead to poor performance of the controlled system, or even instability if they are not taken into account in the design process. The first solution to explicitly compensate delays was proposed in 1959 with the introduction of the Smith Predictor (SP) [2]. The original SP was formulated in frequency domain, applicable only to SISO open-loop stable plants, and exhibited poor robustness with respect to uncertain parameters $[3,4]$. Over the last decades, many modifications to the SP have been proposed to overcome these limitations, see for example [5, 6, 7] and the references therein.

The same underlying idea of the SP was proposed in time domain for general MIMO stable/unstable systems, introducing the so-called state predictor [8], also known as the reductionbased approach [9]. The robustness of this technique to bounded parametric uncertainties was analyzed later in [10], and extended to unknown constant and time-varying delays in [11] and [12], respectively. Complementary results have been obtained in the discrete-time framework

*Correspondence to: Ricardo Sanz, Instituto de Automática e Informática Industrial, Universitat Politècnica de València, 46022 Spain; E-mail: risanzdi@gmail.com 
$[13,14,15]$, and also for nonlinear systems $[16,17,18,19,20]$, even when the plant is unstable. The aforementioned works were focused on proving the closed-loop stability. For linear systems, some researchers have been actively working on reducing the conservatism of the robust stability conditions [21, 22, 23], mainly formulated in terms of linear matrix inequalities (LMIs) [24]. In any case additional robust control structures were applied to improve robustness (which was a common practice in frequency-based methods). Works in this line are scattered. Regarding disturbance rejection, in [25] a low-pass filtered prediction is shown to improve disturbance rejection capabilities and, in [26], better disturbance rejection is achieved by introducing a modified state prediction. Regarding robustness improvement, which is problem this paper is concerned with, a sliding mode control with delay compensation is proposed in [27] to deal with matched uncertainties; in [28], adaptive schemes are introduced to estimate uncertain plant parameters and input delay; and a control strategy is proposed in [29] to deal with Euler-Lagrange-like nonlinear systems with time-varying input delay.

In this paper, the disturbance observer based control (DOBC) is adopted to improve the robustness of the state predictor. The basic idea behind this technique is to use a model of the system along with its input/output information to identify uncertainties and disturbances [30]. There are slightly different techniques that pursue the same goal, which have been recently summarized and explained in [31] (see also the references therein). Most of them make use of some sort of low-pass filter which is needed to obtain a realizable control law. In general, the bandwidth of this filter is desired to be as high as possible to estimate uncertainties in a wide range of frequencies. However, in [32], by means of the Bode's integral formula, it is shown that there is a severe limitation in the choice of the bandwidth if the plant includes time delay. Intuitively, the uncertainties can be estimated arbitrarily fast, but they cannot be counteracted in the same way. This inconvenient can be seen as analogous to the need of detuning PID controllers for open-loop unstable systems [33]. An attempt of combining the state predictor with a DOBC technique known as the ADRC (active disturbance rejection control) can be found in [34], but neither the analysis nor the design problems are tackled, presenting only numerical case studies. Also, a similar idea was explored in [35], where work in [36] is extended to the case of state delays.

This work introduces a new approach to control input-delayed systems, in which the state predictor is modified and combined with an uncertainty estimator. The uncertain part of the model, possibly nonlinear, is neglected when designing the controller, and counteracted by using the information provided by the uncertainty estimator. Furthermore, the conventional state prediction is modified to include also this information. The closed-loop is analyzed using Lyapunov-Krasovskii functionals. A computable robust stability criterion is proved first in Theorem 1, which is cast into a minimization problem constrained to a linear matrix inequality (LMI). Additionally, for a given input delay, an Iterative-LMI (ILMI) algorithm is proposed to design stabilizing tuning parameters in Theorem 2. Once solved, it yields a feedback gain matrix, an observer bandwidth and a robustness index, which ensure stability for a prescribed upper bounded delay.

The rest of the paper is structured as follows. In Section 2, the problem formulation and the assumptions are presented. The building blocks of the proposed control structure are introduced in Section 3 where the closed-loop equations are also derived. The main results are shown in Section 4. Finally, the results are illustrated with a numerical example in Section 5.

Notation: The $n$-dimensional Euclidean space is denoted by $\mathbb{R}^{n}$ while $\mathbb{R}^{n \times m}$ is the set of $n \times m$ real matrices, and $I$ is the identity matrix of appropriate dimensions. The notation $X>0(X \geq 0)$ means that $X \in \mathbb{R}^{n \times n}$ is a real positive definite (semi-definite) matrix. The standard Euclidean vector norm as well as its induced matrix norm are represented by $\|\cdot\|$. The function $\phi(s): \mathbb{R}_{\geq 0} \rightarrow \mathbb{R}$ is said to belong to $L_{1}[0, \infty)$ if the norm $\|\phi(t)\|_{1}=\int_{0}^{\infty}|\phi(s)| \mathrm{d} s$ exists and is finite. 


\section{PROBLEM FORMULATION AND PRELIMINARIES}

Consider the class of multivariable nonlinear input-delay systems represented by

$$
\begin{aligned}
& \dot{x}(t)=A x(t)+B[u(t-h)+w(x)] \\
& u(s)=u_{0}(s) \quad s \in[-h, 0) \\
& x(0)=x_{0}
\end{aligned}
$$

where $x(t) \in \mathbb{R}^{n}$ is the state, $u(t) \in \mathbb{R}^{m}$ is the control, $A \in \mathbb{R}^{n \times n}, B \in \mathbb{R}^{n \times m}$ are known matrices, $h$ is a known constant input delay, $w: \mathcal{D} \rightarrow \mathbb{R}^{m}$ is an unknown (possibly nonlinear) function, and $\mathcal{D} \subset \mathbb{R}^{n}$ is an open connected set that contains the origin $x=0$. The source of a system such as the one described by (1) could be a more general nonlinear system after an appropriate change of coordinates [37]. The system defined in (1) implies that the uncertain term $B w(x(t))$ is matched, that is, it belongs to the range space of the matrix $B$, which is a rather conventional assumption in robust control problems [31].

\section{Assumption 1}

The pair $(A, B)$ is stabilizable

\section{Assumption 2}

The function $w(x)$ : i.) vanishes at the origin; ii.) is differentiable on $\mathcal{D}$ with derivative locally bounded by $\nabla w_{x}^{T} \nabla w_{x} \leq \beta^{2} H^{T} H$ for some $H \in \mathbb{R}^{m \times n}, \beta \geq 0$

The Assumption 1 is standard in analysis of both nonlinear and time-delay systems, as it implies the existence of a Lyapunov function for the nominal non-delayed closed-loop system $\dot{\mathcal{X}}=(A+B K) \mathcal{X}(t)$. This is necessary because the analysis techniques used throughout this paper to deal with delays and uncertainties require the stability of the nominal system. The Assumption 2 is slightly stronger than Lipschitz continuity (the latter allows also functions that are differentiable almost everywhere). The matrix $H$ can be useful to describe how the uncertainty affects each of the states and thus reduce conservatism in the stability conditions.

\section{PROPOSED CONTROL STRATEGY}

The building blocks of the proposed control strategy are presented in this section. The uncertainty estimator for time-delay systems is derived first, whose output is denoted by $\hat{w}(t)$. The novel state prediction $\hat{x}(t+h)$ that includes information about the uncertainty estimation is introduced next. Finally, both elements are combined into a composite control law and the closed-loop equations are derived.

\subsection{Uncertainty estimation}

The uncertainty estimation is obtained by constructing a reduced-order observer from the system model (1). The observer derived next is an adaptation of the one proposed in [38]. Note that the unknown term can be obtained from (1) as $w(x(t))=B^{+}[\dot{x}(t)-A x(t)-B u(t-h)]$, where $B^{+}=\left(B^{T} B\right)^{-1} B^{T}$ is the pseudoinverse of the matrix $B$. However, the previous equation cannot be computed because the state derivative $\dot{x}(t)$ is not accessible. To circumvent this issue, a filtered estimation $\hat{w}(t) \in \mathbb{R}^{m}$ is proposed such that

$$
\dot{\hat{w}}(t) \triangleq-\Omega \hat{w}(t)+\Omega B^{+}[\dot{x}(t)-A x(t)-B u(t-h)]
$$

where $\Omega \in \mathbb{R}^{m \times m}$ is a positive definite diagonal matrix $\Omega \triangleq \operatorname{diag}\left\{\omega_{1}, \omega_{2}, \ldots, \omega_{m}\right\}$, with $\omega_{j}>0$. In this way, the explicit state derivative $\dot{x}(t)$ can be removed from the observer by performing the change of variable $\hat{\xi}(t) \triangleq \hat{w}(t)-\Omega B^{+} x(t)$ in (2), which leads to the following reduced-order observer

$$
\begin{aligned}
\dot{\hat{\xi}}(t) & =-\Omega \hat{\xi}(t)-\left(\Omega^{2} B^{+}+\Omega B^{+} A\right) x(t)-\Omega u(t-h) \\
\hat{w}(t) & =\hat{\xi}(t)+\Omega B^{+} x(t)
\end{aligned}
$$




\section{Remark 1}

Notice that the observer (3)-(4) is computed using only information from the actual state $x(t)$, and the past control input $u(t-h)$. The Equation (2) can be seen as a low-pass filter with $m$ channels, driven by the virtual input $w(x(t))=B^{+}[\dot{x}(t)-A x(t)-B u(t-h)]$, and hence each parameter $\omega_{j}$ should be understood as the bandwidth of the $j$-th channel.

The error between the actual uncertainty and its estimation is defined as

$$
e(t) \triangleq w(x(t))-\hat{w}(t)
$$

Differentiating (4), plugging (3) in, rearranging terms and using the system model (1), the following expression is obtained

$$
\dot{\hat{w}}(t)=-\Omega \hat{w}(t)+\Omega w(x(t))
$$

The fact that $\hat{w}(t)$ is a low-passed filtered estimation of $w(x(t))$, pointed out in Remark 1 , is highlighted by (6). Now, it is straightforward to use (6) and (5) to obtain the dynamics of the estimation error as

$$
\dot{e}(t)=-\Omega e(t)+\dot{w}(x(t))
$$

\section{Remark 2}

From (7), it can be seen that the estimation error decays, assuming $\dot{w}(x(t))$ bounded, with an exponential rate $\alpha=\lambda_{\max }(\Omega)=\max _{j} \omega_{j}$. Therefore, one would be interested in choosing the bandwidths $\omega_{j}$ as large as possible.

So far, the input delay does not play any role as it does not affect the uncertainty estimation. However, it is critical when attempting to cancel out its effect in the closed-loop. In contrast to the estimation error $e(t)$, the cancellation error is defined as

$$
\sigma(t) \triangleq w(x(t))-\hat{w}(t-h)
$$

which will naturally arise later in the closed-loop equations.

\subsection{Predictor-based feedback}

For LTI time delay systems $\dot{\mathcal{X}}(t)=A \mathcal{X}(t)+B \mathcal{U}(t-h)$, the conventional predictive feedback $\mathcal{U}(t)=\mathcal{K} \hat{\mathcal{X}}(t+h)$, with $\hat{\mathcal{X}}(t+h)=e^{A h} \mathcal{X}(t)+\int_{-h}^{0} e^{-A s} B \mathcal{U}(t+s) \mathrm{d} s$ is widely known in the literature as the finite spectrum assignment (FSA), as it renders (in the nominal case) a closed-loop with a finite dimensional characteristic polynomial, for any arbitrarily large delay $h \geq 0$.

In this paper, a new state prediction is introduced that includes additional information about the estimated uncertainty $\hat{w}(t)$. Such prediction is defined by

$$
\hat{x}(t+h) \triangleq e^{A h} x(t)+\int_{-h}^{0} e^{-A s} B[u(t+s)+\hat{w}(t+s)] \mathrm{d} s
$$

Notice that the exact prediction of the state from $t$ to $t+h$ can be obtained from (1) as

$$
x(t+h)=e^{A h} x(t)+\int_{-h}^{0} e^{-A s} B[u(t+s)+w(x(t+s+h))] \mathrm{d} s
$$

Of course, (10) cannot be computed because $w(x(t))$ is unknown. Using (9)-(10) and the definition (8), the error between the exact and the proposed prediction is given by

$$
x(t+h)-\hat{x}(t+h)=\int_{-h}^{0} e^{-A s} B \sigma(t+s+h) \mathrm{d} s
$$




\subsection{Control law}

The state prediction and the uncertainty estimation are brought together into a composite control law defined by

$$
u(t)=K \hat{x}(t+h)-\hat{w}(t),
$$

with $K \in \mathbb{R}^{m \times n}$. The predicted feedback is used to stabilize the nominal part of the system and compensate for the delay, while the estimation $\hat{w}(t)$ is fed back to the system in order to cancel out the effect of the uncertainties. Notice that this is a rather conventional approach when attenuating matched uncertainties [31].

\subsection{Closed-loop equations}

Using the control law (12) and (11) into the system (1) yields the following closed-loop dynamics

$$
\dot{x}(t)=(A+B K) x(t)+B \sigma(t)+B \int_{-h}^{0} G(s) \sigma(t+s) \mathrm{d} s
$$

where $G(s)=-K e^{-A s} B \in \mathbb{R}^{m \times m}$ has been defined. As mentioned above, the cancellation error $\sigma(t)$, arises in the closed-loop equation due to the input delay. The dynamics of the cancellation error is obtained by differentiating (8), using (6) evaluated at $t-h$, and adding and subtracting $\Omega w(x(t))$, which yields

$$
\dot{\sigma}(t)=-\Omega \sigma(t)+\Omega[w(x(t))-w(x(t-h))]+\dot{w}(x, t)
$$

Now, for analysis purposes, it is convenient to get rid of the of the term $[w(x(t))-w(x(t-h))]$ in (14), so that the entire system is driven only by $\dot{w}(x, t)$. To do so, the estimation error dynamics (7) is integrated and used to rewrite

$$
w(x(t))-w(x(t-h))=e(t)-e(t-h)+\Omega \int_{-h}^{0} e(t+s) \mathrm{d} s
$$

Let us denote by $g_{i j}(s)$ the $(i, j)$-entry of $G(s)$, which can be expressed as a sum of scalar functions as $G(s)=\sum_{i=1}^{m} \sum_{j=1}^{m} \delta_{i j} g_{i j}(s)$, with $\delta_{i j} \in \mathbb{R}^{m \times m}$ a matrix such that its $(i, j)$-entry is equal one while the rest are zero. This formulation is chosen so that the remaining kernel in the distributed delay is scalar. Defining the augmented state vector $\eta(t) \triangleq\left[x^{T}(t), \sigma^{T}(t), e^{T}(t)\right]^{T}$ and gathering (7), (13)-(15) leads to

$$
\begin{aligned}
\dot{\eta}(t) & =\left[\begin{array}{ccc}
A+B K & B & 0 \\
0 & -\Omega & \Omega \\
0 & 0 & -\Omega
\end{array}\right] \eta(t)+\sum_{i=1}^{m} \sum_{j=1}^{m}\left[\begin{array}{ccc}
0 & B \delta_{i j} & 0 \\
0 & 0 & 0 \\
0 & 0 & 0
\end{array}\right] \int_{-h}^{0} g_{i j}(s) \eta(t+s) \mathrm{d} s \\
& +\int_{-h}^{0}\left[\begin{array}{ccc}
0 & 0 & 0 \\
0 & 0 & \Omega^{2} \\
0 & 0 & 0
\end{array}\right] \eta(t+s) \mathrm{d} s+\left[\begin{array}{ccc}
0 & 0 & 0 \\
0 & 0 & -\Omega \\
0 & 0 & 0
\end{array}\right] \eta(t-h)+\left[\begin{array}{c}
0 \\
I_{m} \\
I_{m}
\end{array}\right] \dot{w}(x)
\end{aligned}
$$

The system (16) is the result of controlling (1) with (3)-(4), (9) and (12). In what follows, a practically computable criterion to check the robust stability of (16) is derived, in the form of a minimization problem subject to LMI constraints (Theorem 1). Additionally, for a given input delay, an Iterative-LMI (ILMI) algorithm is proposed to design stabilizing tuning parameters $K$ and $\Omega$ (Theorem 2). 


\section{MAIN RESULTS}

In this section, sufficient stability conditions for the closed-loop stability based on LyapunovKrasovskii functionals are derived. In order to reduce conservatism, the system (16) is transformed into a descriptor form [39]. The terms $\eta(t-h)$ are replaced by $\eta(t)-\int_{-h}^{0} \mu(t+s) \mathrm{d} s$ using the Newton-Leibnitz formula, which leads to

$$
\begin{aligned}
\dot{\eta}(t) & =\mu(t) \\
0 & =-\mu(t)+A_{1} \eta(t)+A_{2} \int_{-h}^{0} \eta(t+s) \mathrm{d} s+A_{3} \int_{-h}^{0} \mu(t+s) \mathrm{d} s \\
& +\sum_{i=1}^{m} \sum_{j=1}^{m} A_{4_{i j}} \int_{-h}^{0} g_{i j}(s) \eta(t+s) \mathrm{d} s+\Gamma \dot{w}(x)
\end{aligned}
$$

with

$$
\begin{gathered}
A_{1}=\left[\begin{array}{ccc}
A+B K & B & 0 \\
0 & -\Omega & 0 \\
0 & 0 & -\Omega
\end{array}\right] \quad A_{2}=\left[\begin{array}{ccc}
0 & 0 & 0 \\
0 & 0 & \Omega^{2} \\
0 & 0 & 0
\end{array}\right] \quad A_{3}=\left[\begin{array}{ccc}
0 & 0 & 0 \\
0 & 0 & \Omega \\
0 & 0 & 0
\end{array}\right] \\
A_{4_{i j}}=\left[\begin{array}{ccc}
0 & B \delta_{i j} & 0 \\
0 & 0 & 0 \\
0 & 0 & 0
\end{array}\right] \quad \Gamma=\left[\begin{array}{c}
0 \\
I_{m} \\
I_{m}
\end{array}\right]
\end{gathered}
$$

Theorem 1

Consider some prescribed tuning for the controller parameters $K$ and $\Omega$. Under the Assumptions 1 and 2, the system (1) controlled with (3)-(4), (9) and (12) is robustly asymptotically stable for any $0 \leq \beta \leq \beta^{*} \triangleq\left(\gamma^{*}\right)^{1 / 2}$ and any delay $0 \leq h \leq \bar{h}$, if there exist symmetric positive definite matrices $P_{1}, R, S, W_{i j} \in \mathbb{R}^{(n+2 m) \times(n+2 m)}$ and real matrices $N_{2}, N_{3} \in \mathbb{R}^{(n+2 m) \times(n+2 m)}$ such that the following problem is feasible

$$
\begin{aligned}
\gamma^{*}=\underset{\gamma>0}{\operatorname{argmin}} & \gamma \\
& \text { subject to } \Psi^{0}<0
\end{aligned}
$$

with

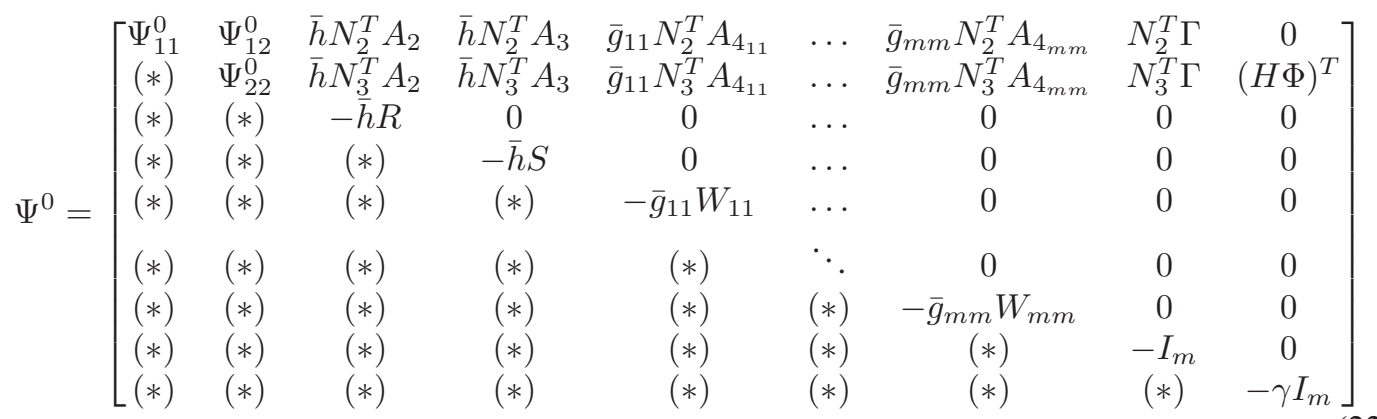

$$
\begin{aligned}
& \Psi_{11}^{0}=N_{2}^{T} A_{1}+A_{1}^{T} N_{2}+\bar{h} R+\sum_{i=1}^{m} \sum_{j=1}^{m} \bar{g}_{i j} W_{i j} \quad \bar{g}_{i j}=\int_{-\bar{h}}^{0}\left|g_{i j}(s)\right| \mathrm{d} s \\
& \Psi_{12}^{0}=P_{1}-N_{2}^{T}+A_{1}^{T} N_{3} \quad \Phi=\left[I_{n}, 0_{n \times m}, 0_{n \times m}\right] \\
& \Psi_{22}^{0}=-N_{3}-N_{3}^{T}+\bar{h} S
\end{aligned}
$$

Proof

See Appendix A. 


\section{Theorem 2}

Under the Assumptions 1-2, the system (1) can be robustly asymptotically stabilized with (3)-(4), (9) and (12), for any $0 \leq \beta \leq\left(\gamma^{*}\right)^{-1 / 2}$ and any delay $0 \leq h \leq \bar{h}$, if there exist symmetric positive definite matrices $Y_{1}, Y_{R}, Y_{S}, Y_{W_{i j}} \in \mathbb{R}^{(n+2 m) \times(n+2 m)}$, positive scalars $\gamma, \kappa_{x}^{(0)}, \ldots, \kappa_{x}^{(m)}, \kappa_{y}^{(0)}, \ldots, \kappa_{y}^{(m)} \in \mathbb{R}_{>0}$, adjustable weights $\lambda_{j}$, a tuning parameter $\epsilon \in \mathbb{R}$, and block-diagonal matrices $Y_{2}=\operatorname{diag}\left\{Y_{2}^{(0)}, Y_{2}^{(1)}, \ldots, Y_{2}^{(m)}, Y_{2}^{(1)}, \ldots, Y_{2}^{(m)}\right\}$, $X=\operatorname{diag}\left\{X^{(0)}, X^{(1)}, \ldots, X^{(m)}, X^{(1)}, \ldots, X^{(m)}\right\}$, with a real matrix $X^{(0)} \in \mathbb{R}^{n \times m}$, a positive definite matrix $Y_{2}^{(0)} \in \mathbb{R}^{n \times n}$, positive scalars $Y_{2}^{(1)}, \ldots, Y_{2}^{(m)} \in \mathbb{R}_{>0}$, and scalars $X^{(1)}, \ldots, X^{(m)} \in \mathbb{R}$, such that the following problem is feasible

$$
\begin{aligned}
\gamma^{*}=\underset{\gamma>0}{\operatorname{argmin}} & \lambda_{\gamma} \gamma+\sum_{j=0}^{m} \lambda_{j}\left(\kappa_{y}^{(j)}+\kappa_{x}^{(j)}\right) \\
\text { subject to } & \left\{\begin{array}{c}
\Psi<0 \\
{\left[\begin{array}{cc}
-\kappa_{x}^{(j)} I & \left(X^{(j)}\right)^{T} \\
(*) & -I
\end{array}\right]<0, \quad\left[\begin{array}{cc}
Y_{2}^{(j)} & I \\
(*) & \kappa_{y}^{(j)}
\end{array}\right]>0, \quad j=1, \ldots, m}
\end{array}\right.
\end{aligned}
$$

with

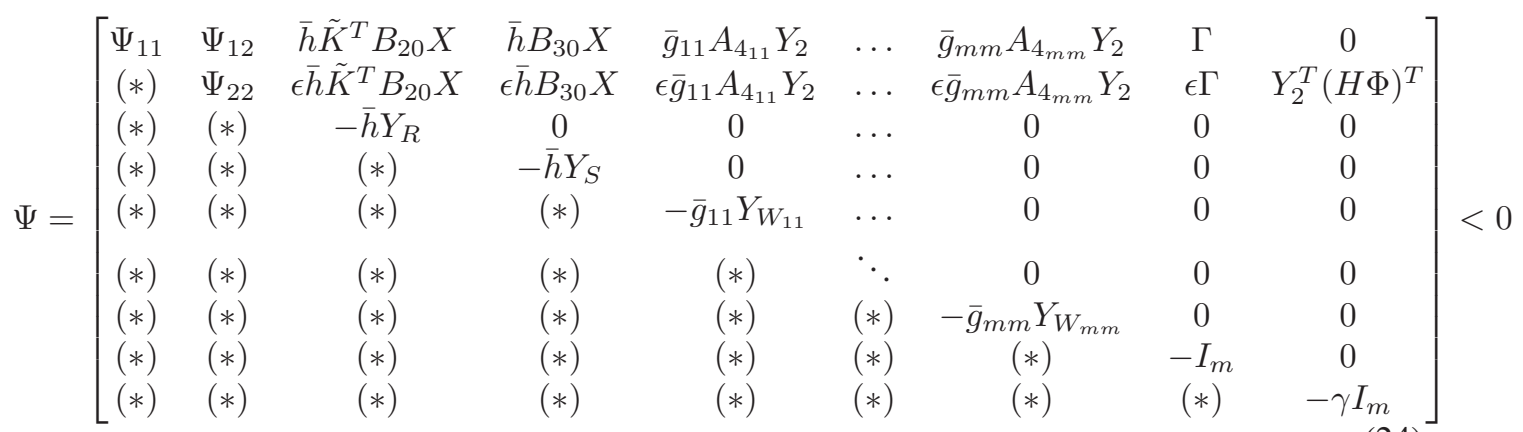

$$
\begin{array}{ll}
\Psi_{11}=A_{10} Y_{2}+B_{10} X+X^{T} B_{10}^{T}+Y_{2}^{T} A_{10}^{T}+\bar{h} Y_{R}+\sum_{i=1}^{m} \sum_{j=1}^{m} \bar{g}_{i j} Y_{W_{i j}} & \bar{g}_{i j}=\int_{-\bar{h}}^{0}\left|g_{i j}(s)\right| \mathrm{d} s \\
\Psi_{12}=Y_{1}-Y_{2}+\epsilon\left(X^{T} B_{10}^{T}+Y_{2}^{T} A_{10}^{T}\right) & \Phi=\left[I_{n}, 0_{n \times m}, 0_{n \times m}\right] \\
\Psi_{22}=-\epsilon\left(Y_{2}^{T}-Y_{2}\right)+\bar{h} Y_{S} &
\end{array}
$$

$$
A_{10}=\left[\begin{array}{ccc}
A & B & 0 \\
0 & 0 & 0 \\
0 & 0 & 0
\end{array}\right] \quad B_{10}=\left[\begin{array}{ccc}
B & 0 & 0 \\
0 & -I_{m} & 0 \\
0 & 0 & -I_{m}
\end{array}\right] \quad B_{20}=B_{30}=\left[\begin{array}{ccc}
0 & 0 & 0 \\
0 & 0 & I_{m} \\
0 & 0 & 0
\end{array}\right]
$$

and $\tilde{K}=\operatorname{diag}\{K, \Omega, \Omega\}$. Furthermore, the stabilizing feedback control gain is given by $K=X^{(0)}\left(Y_{2}^{(0)}\right)^{-1}$ and each of the observer bandwidths is given by $\omega_{j}=X^{(j)}\left(Y_{2}^{(j)}\right)^{-1}$, satisfying $\|K\|<\kappa_{y}^{(0)} \sqrt{\kappa_{x}^{(0)}}$ and $\left|\omega_{j}\right|<\kappa_{y}^{(j)} \sqrt{\kappa_{x}^{(j)}}$, respectively.

\section{Proof}

See Appendix B.

The matrix $\tilde{K}$, which is an output of the minimization problem (23), appears in (24), and thus the problem has to be solved by iterating. Notice that (24) becomes a standard LMI if $\tilde{K}$ is fixed. The following Iterative-LMI algorithm, similar to [40], is used to solve this problem. Step 1: Choose $\tilde{K}_{0}=0$ initially and let $k=1$. Step 2: Solve the optimization problem (23) with $\tilde{K}=\tilde{K}_{k-1}$ to obtain $X_{k}, Y_{2_{k}}$ and compute $\tilde{K}_{k}=X_{k} Y_{2_{k}}^{-1}$. Step 3: If $\left\|\tilde{K}_{k}-\tilde{K}_{k-1}\right\|<\delta$ for some sufficiently small $\delta>0$, or the maximum number of iterations is reached, then stop. Otherwise, let $k \rightarrow k+1$ and go to Step 2. 


\section{Remark 3}

The weights in the minimization problem (23) can be adjusted to reach a trade-off between robustness, feedback gain norm, and observer bandwidth (in practice, if measurements are noisy, having a low observer bandwidth is convenient). Furthermore, there is another user-supplied parameter, $\epsilon$, which arises when manipulating the matrix inequality. The optimal value of this parameter can be easily found using by numerical optimization because the cost exhibits a convex behavior with respect to $\epsilon$, as explained in Remark 5 of [21]. This fact is demonstrated below using a numerical example and illustrated in Fig. 1.c.

\section{NUMERICAL EXAMPLE}

In order to illustrate the proposed strategy, an uncertain second-order system with delayed input $\ddot{y}(t)=u(t-h)+w(y, \dot{y})$ is chosen, which can be expressed in the form of (1) with

$$
A=\left[\begin{array}{ll}
0 & 1 \\
0 & 0
\end{array}\right] \quad B=\left[\begin{array}{l}
0 \\
1
\end{array}\right]
$$

and $x=\left[x_{1}, x_{2}\right]^{T}$ and $x_{1}=y, x_{2}=\dot{y}$.

\subsection{Linear uncertainty}

Let us consider a long time delay $h=1 \mathrm{~s}$, and a linear uncertainty $w(x)=\beta x_{1}$. Since $w(x)$ is linear, its gradient does not depend on $x$ and it is simply given by $\nabla w=[\beta, 0]$. Assumption 2 is then satisfied globally with $H=[1,0]$. In this case, a controller designed by means of Theorem 2 will ensure global stability if $\beta<\beta^{*}$.

Solving the minimization problem (23) in Theorem 2 with weights ${ }^{\dagger} \lambda_{\gamma}=0.5, \lambda_{0}=\lambda_{1}=0.25$ yields an optimal $\gamma^{*}=189.6$ and matrices

$$
\begin{gathered}
Y_{2}=\left[\begin{array}{cccc}
373.59 & -51.08 & 0 & 0 \\
-51.08 & 47.98 & 0 & 0 \\
0 & 0 & 6.14 & 0 \\
0 & 0 & 0 & 6.14
\end{array}\right] \quad X=\left[\begin{array}{ccccc}
-17.64 & -65.43 & 0 & 0 \\
0 & 0 & 3.96 & 0 \\
0 & 0 & 0 & 3.96
\end{array}\right] \\
\tilde{K}=X\left(Y_{2}\right)^{-1}=\left[\begin{array}{cccc}
-0.27 & -1.65 & 0 & 0 \\
0 & 0 & 0.65 & 0 \\
0 & 0 & 0 & 0.65
\end{array}\right]
\end{gathered}
$$

Therefore, the controller parameters are $K=[-0.27,-1.65]$ and $\omega_{1}=0.65 \mathrm{rad} / \mathrm{s}$, and the maximum allowable uncertainty is $\beta^{*}=\left(\gamma^{*}\right)^{-1 / 2}=0.073$. Simulation results are presented in Fig. 1, showing both nominal and robust responses in Figs. 1.a and 1.b, respectively. The convex behavior of the cost with respect to the parameter $\epsilon$ is also depicted in Fig. 1.c. In this case, an optimal value of $\epsilon=1.2$ is found. As an example, for that value of $\epsilon$, the history of the convergence criterion $\left\|\tilde{K}_{k}-\tilde{K}_{k-1}\right\|$ can be seen in Fig. 1.d, which is stopped at $k=20$.

The decomposition process used in Theorem 2 introduces some conservatism in the resulting robustness index. Using Theorem 1, an almost four times larger value $\beta^{*}=0.27$ is obtained. It can be seen in Fig. 2 that this value is quite close to the actual stability limit.

Among the existing references, very few of them follow the idea of combining predictive feedback with an additional robustifying structure. The approach presented in [26] is suitable for a numerical comparison, as it is a slight modification that improves the conventional predictive feedback [8]. A simulation in the same scenario as above shows that the proposed controller can keep the system

\footnotetext{
${ }^{\dagger}$ Since (23) is a multi-objective minimization problem, it is reasonable to choose the weights such that $\lambda_{\gamma}+\sum_{j} \lambda_{j}=1$. This a simple method that transforms the problem into a single-objective minimization, where the weights determine the relative importance of each objective function with respect to each other [41].
} 

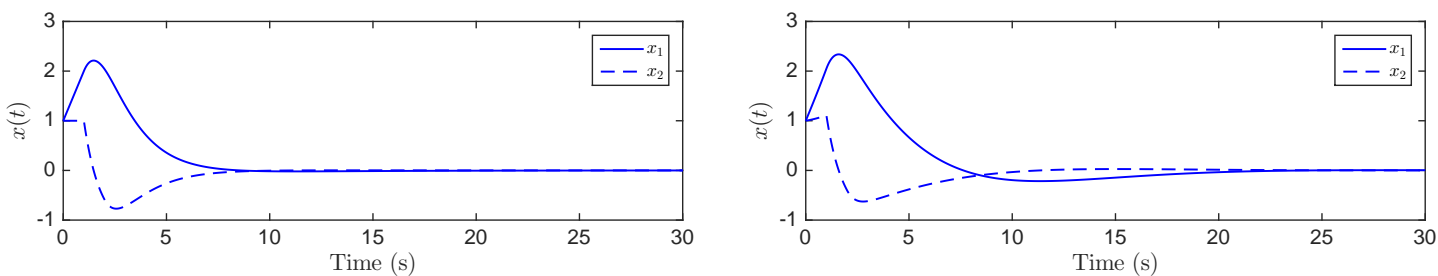

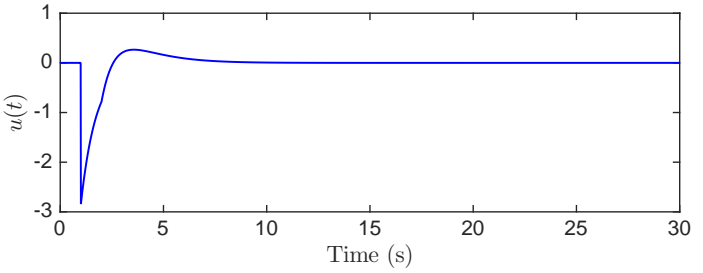

(a) Nominal response, $\beta=0$

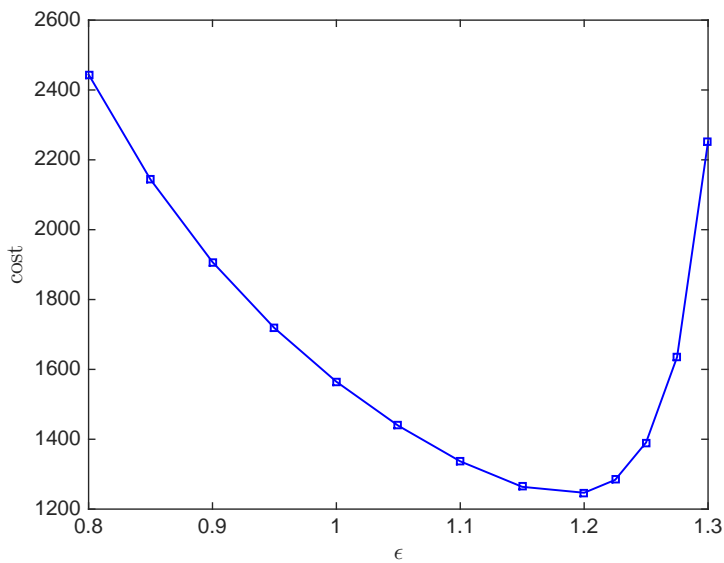

(c) Convex behavior of the cost w.r.t. the parameter $\epsilon$

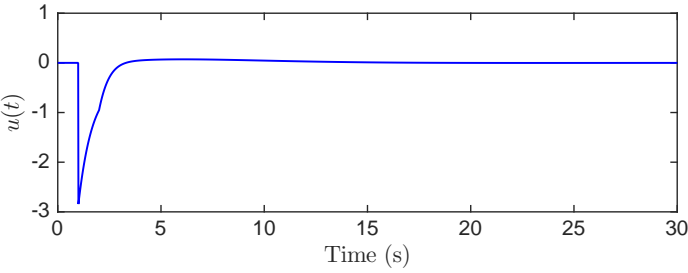

(b) Robust response, $\beta=0.07$

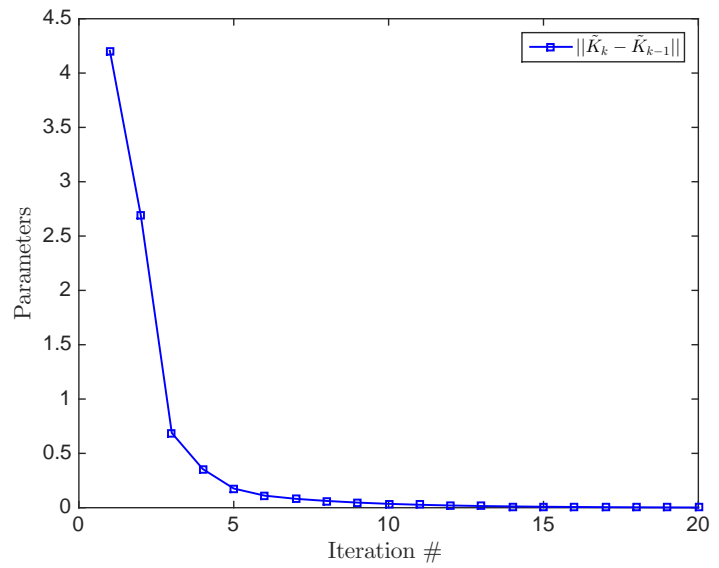

(d) Example of convergence history

Figure 1. Simulations with linear uncertainty $w(x)=\beta x_{1}$, the system starting at $x(0)=[1,1]^{T}$, input delay $h=1 \mathrm{~s}$, and controller parameters $K=[-0.27,-1.65]$ and $\omega_{1}=0.65 \mathrm{rad} / \mathrm{s}$, designed by means of Theorem 2

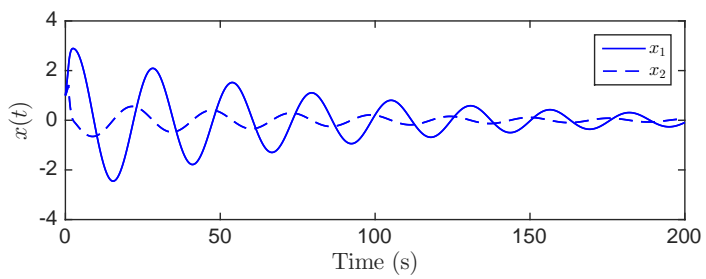

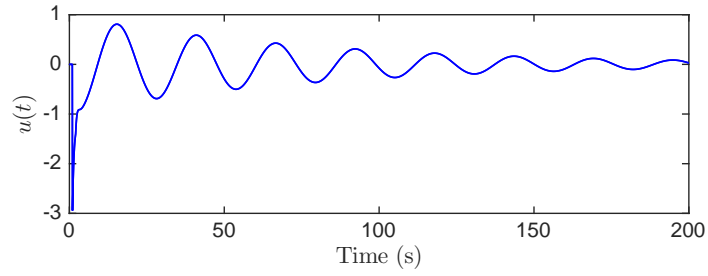

(a) Robust response, $\beta=0.27$

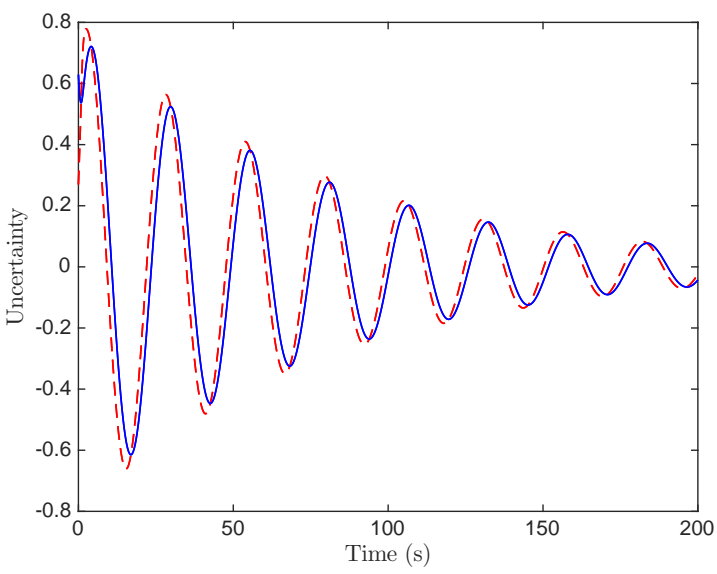

(b) Uncertainty estimation

Figure 2. Validation of the robust stability bound $\beta=0.27$ obtained by means of Theorem 1 
stable while the solutions presented in [8], [26] cannot. Therefore, the proposed scheme can improve the robustness of the closed-loop system.

\subsection{Nonlinearity}

Let us consider now a smaller input delay $h=0.2 \mathrm{~s}$ and a nonlinearity $w(x)=\sin \left(x_{1}+x_{2}\right)$. Its gradient is obtained as

$$
\nabla w(x)=\left[\cos \left(x_{1}+x_{2}\right), \cos \left(x_{1}+x_{2}\right)\right] \leq[1,1]
$$

and thus Assumption 2 is satisfied globally with $H=[1,1]$ and $\beta=1$. Because of the properties of this particular nonlinearity, a controller designed by means of Theorem 2 will ensure global stability if $\beta^{*}>1$, and only local stability if $\beta^{*} \leq 1$. Using Theorem 2 with weights $\lambda_{\gamma}=0.8, \lambda_{0}=\lambda_{1}=0.1$, yields an optimal $\beta^{*}=\left(\gamma^{*}\right)^{-1 / 2}=1.17$ and controller parameters $K=[-1.19,-2.93], \omega_{1}=1.89 \mathrm{rad} / \mathrm{s}$. From the reasoning above, this controller should be globally

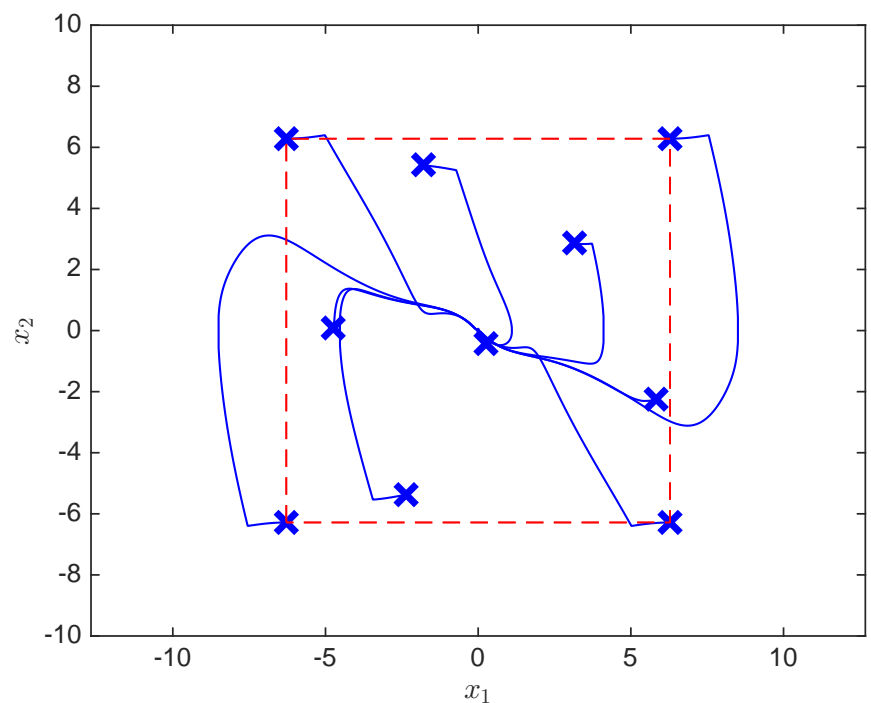

Figure 3. Validation of Theorem 2 in the case of nonlinear uncertainties
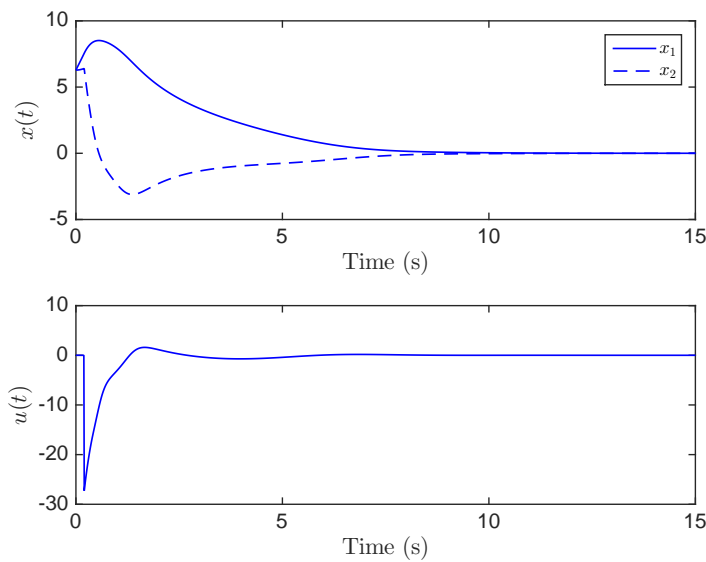

(a) State evolution and control input

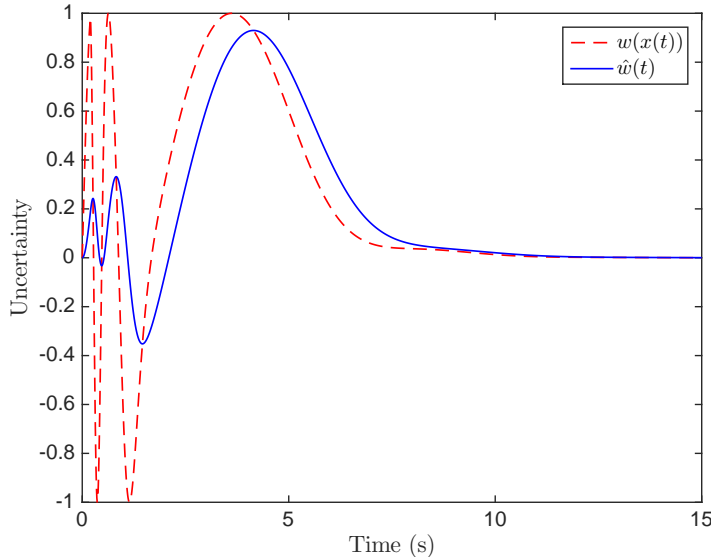

(b) Uncertainty estimation

Figure 4. Simulations with a nonlinearity $w(x)=\sin \left(x_{1}+x_{2}\right)$, the system starting at $x(0)=[2 \pi, 2 \pi]^{T}$, input delay $h=0.2 \mathrm{~s}$, and controller parameters $K=[-1.19,-2.93]$ and $\omega_{1}=1.89 \mathrm{rad} / \mathrm{s}$, designed by means of Theorem 2 
stabilizing in spite of the nonlinearity. The global stability is verified by running a set of ten simulations starting within $x(0) \in[-2 \pi, 2 \pi] \times[-2 \pi, 2 \pi]$ as shown in Fig. 3. To show just one example, the state trajectory starting from $x(0)=[2 \pi, 2 \pi]^{T}$ is depicted in Fig. 4.a and the corresponding uncertainty estimation can be seen in Fig. 4.b.

\section{Remark 4}

One of the benefits of the controller synthesis procedure proposed in this paper is that the observer bandwidths are also automatically tuned. In the delay-free case, the bandwidth is simply chosen as small as possible (only limited in practice by the measurement noise and the sampling period). However, if there is input delay, a small filter bandwidth can make the closed-loop unstable [32].

\section{CONCLUSIONS AND FUTURE WORK}

In this paper, a robust control scheme for input-delay systems has been introduced and analyzed. A stability criterion has been derived in terms of a LMI and a design procedure has been proposed, which needs to be solved using an Iterative-LMI algorithm. This control structure is able to deal with matched and possibly nonlinear uncertainties even in the presence of input delays, as it has been shown through numerical examples. Future research will be focused on extending the analysis to prove robustness with respect to time-varying delays or modifying the proposed scheme to deal with unmatched uncertainties.

\section{ACKNOWLEDGEMENTS}

This work was partially supported by projects PROMETEOII/2013/004, Conselleria d'Educació, Generalitat Valenciana, and TIN2014-56158-C4-4-P-AR, Ministerio de Economía y Competitividad, Spain.

\section{A. PROOF OF THEOREM 1}

\section{A.1. Auxiliary lemma: Jensen's inequality with variable kernels}

Lemma $1([42,43])$

Consider an $n \times n$ matrix $W>0$, a scalar function $g:[0, \infty) \rightarrow \mathbb{R}$ belonging to $L_{1}[0, \infty)$ and a vector function $\phi:[0, \infty) \rightarrow \mathbb{R}^{n}$ such that the integrations concerned are well defined. Then, for any scalar $h>0$, the following inequality holds

$$
-\int_{-h}^{0}|g(s)| \phi^{T}(t+s) W \phi(t+s) \mathrm{d} s \leq-\frac{1}{\bar{g}}\left(\int_{-h}^{0} g(s) \phi(t+s) \mathrm{d} s\right)^{T} W\left(\int_{-h}^{0} g(s) \phi(t+s) \mathrm{d} s\right)
$$

where $\bar{g}=\int_{-h}^{0}|g(s)| \mathrm{d} s$.

\section{A.2. Proof of Theorem 1}

Let us choose the Lyapunov candidate function

$$
V(t)=V_{1}(t)+V_{2}(t)+V_{3}(t)+\sum_{i=1}^{m} \sum_{j=1}^{m} V_{4_{i j}}(t)
$$


where

$$
\begin{array}{ll}
V_{1}(t)=\eta^{T}(t) P_{1} \eta(t) & V_{2}(t)=\int_{-h}^{0} \int_{t+s}^{t} \eta^{T}(\tau) R \eta(\tau) \mathrm{d} \tau \mathrm{d} s \\
V_{3}(t)=\int_{-h}^{0} \int_{t+s}^{t} \mu^{T}(\tau) S \mu(\tau) \mathrm{d} \tau \mathrm{d} s & V_{4_{i j}}(t)=\int_{-h}^{0} \int_{t+s}^{t}\left|g_{i j}(s)\right| \eta^{T}(\tau) W_{i j} \eta(\tau) \mathrm{d} \tau \mathrm{d} s
\end{array}
$$

Using Lemma 1, the derivative of $V_{4_{i j}}(t)$ is bounded by

$$
\begin{aligned}
\dot{V}_{4_{i j}}(t) & =\eta^{T}(t)\left[\bar{g}_{i j} W_{i j}\right] \eta(t)-\int_{-h}^{0}\left|g_{i j}(s)\right| \eta^{T}(t+s) W_{i j} \eta(t+s) \mathrm{d} s \\
& \leq \eta^{T}(t)\left[\bar{g}_{i j} W_{i j}\right] \eta(t)+\left(\frac{1}{\bar{g}_{i j}} \int_{-h}^{0} g_{i j}(s) \eta(t+s) \mathrm{d} s\right)^{T}\left[-\bar{g}_{i j} W_{i j}\right]\left(\frac{1}{\bar{g}_{i j}} \int_{-h}^{0} g_{i j}(s) \eta(t+s) \mathrm{d} s\right)
\end{aligned}
$$

Similarly, the derivatives of $V_{2}(t)$ and $V_{3}(t)$ are bounded (using Lemma 1 with $g(s)=1$ ) by

$$
\begin{gathered}
\dot{V}_{2}(t) \leq \eta^{T}(t) h R \eta(t)+\left(\frac{1}{h} \int_{-h}^{0} \eta^{T}(t+s) \mathrm{d} s\right)[-h R]\left(\frac{1}{h} \int_{-h}^{0} \eta(t+s) \mathrm{d} s\right) \\
\dot{V}_{3}(t) \leq \mu^{T}(t) h S \mu(t)+\left(\frac{1}{h} \int_{-h}^{0} \mu^{T}(t+s) \mathrm{d} s\right)[-h S]\left(\frac{1}{h} \int_{-h}^{0} \mu(s) \mathrm{d} s\right)
\end{gathered}
$$

The derivative of the first contribution is obtained as $\dot{V}_{1}(t)=2 \eta^{T}(t) P_{1} \dot{\eta}(t)$. Using (17)-(18) and the inequalities (28)-(30), it follows that

$$
\begin{aligned}
\dot{V}(t) \leq & 2 \eta^{T}(t) P_{1} \mu(t)+2\left[\eta^{T}(t) N_{2}^{T}+\mu^{T}(t) N_{3}^{T}\right] \cdot[\text { RHS of }(18)]+(28)+(29)+(30) \\
& \leq q^{T}(t)\left[\begin{array}{cccccccc}
(1,1) & (1,2) & h N_{2}^{T} A_{2} & h N_{2}^{T} A_{3} & \bar{g}_{11} N_{2}^{T} A_{4_{11}} & \ldots & \bar{g}_{m m} N_{2}^{T} A_{4_{m m}} & N_{2}^{T} \Gamma \\
(*) & (2,2) & h N_{3}^{T} A_{2} & h N_{3}^{T} A_{3} & \bar{g}_{11} N_{3}^{T} A_{4_{11}} & \ldots & \bar{g}_{m m} N_{3}^{T} A_{4_{m m}} & N_{3}^{T} \Gamma \\
(*) & (*) & -h R & 0 & 0 & \ldots & 0 & 0 \\
(*) & (*) & (*) & -h S & 0 & \ldots & 0 & 0 \\
(*) & (*) & (*) & (*) & -\bar{g}_{11} W_{11} & \ldots & 0 & 0 \\
(*) & (*) & (*) & (*) & (*) & \ddots & 0 & 0 \\
(*) & (*) & (*) & (*) & (*) & (*) & -\bar{g}_{m m} W_{m m} & 0 \\
(*) & (*) & (*) & (*) & (*) & (*) & (*) & 0
\end{array}\right] q(t)
\end{aligned}
$$

where the matrices $N_{2}, N_{3}$ help in reducing conservatism by introducing additional freedom [21], and $(1,1)=N_{2}^{T} A_{1}+A_{1}^{T} N_{2}+h R+\sum_{i=1}^{m} \sum_{j=1}^{m} \bar{g}_{i j} W_{i j}, \quad(1,2)=P_{1}-N_{2}^{T}+A_{1}^{T} N_{3}, \quad(2,2)=$ $-N_{3}-N_{3}^{T}+h S$ and

$$
\begin{aligned}
q_{0}(t) \triangleq & \operatorname{col}\left\{\eta(t), \mu(t), \frac{1}{h} \int_{-h}^{0} \eta(t+s) \mathrm{d} s, \frac{1}{h} \int_{-h}^{0} \mu(t+s) \mathrm{d} s, \frac{1}{\bar{g}_{11}} \int_{-h}^{0} g_{11}(s) \eta(t+s) \mathrm{d} s, \ldots,\right. \\
& \left.\frac{1}{\bar{g}_{m m}} \int_{-h}^{0} g_{m m}(s) \eta(t+s) \mathrm{d} s, \dot{w}_{x}(x)\right\}
\end{aligned}
$$

Now, by Assumption 2, one has that $\dot{w}^{T}(x) \dot{w}(x) \leq \dot{x}^{T}(t) H^{T} \beta^{2} H \dot{x}(t)$, which is equivalent to the following quadratic inequality

$$
[\mu(t) \quad \dot{w}(x)]\left[\begin{array}{cc}
(H \Phi)^{T} \beta^{2} H \Phi & 0 \\
0 & -I_{m}
\end{array}\right]\left[\begin{array}{c}
\mu(t) \\
\dot{w}(x)
\end{array}\right] \geq 0
$$

where $\Phi=\left[I_{n}, 0_{n \times m}, 0_{n \times m}\right]$ is defined such that $\dot{x}(t)=\Phi \mu(t)$. Using (31), (32) and the S-procedure [44], it follows that $\dot{V}<0$ is implied by the existence of a positive scalar $\tau>0$ such 
that

$$
\left[\begin{array}{cccccccc}
(1,1) & (1,2) & h N_{2}^{T} A_{2} & h N_{2}^{T} A_{3} & \bar{g}_{11} N_{2}^{T} A_{4_{11}} & \ldots & \bar{g}_{m m} N_{2}^{T} A_{4_{m m}} & N_{2}^{T} \Gamma \\
(*) & (2,2)^{*} & h N_{3}^{T} A_{2} & h N_{3}^{T} A_{3} & \bar{g}_{11} N_{3}^{T} A_{4_{11}} & \ldots & \bar{g}_{m m} N_{3}^{T} A_{4_{m m}} & N_{3}^{T} \Gamma \\
(*) & (*) & -h R & 0 & 0 & \ldots & 0 & 0 \\
(*) & (*) & (*) & -h S & 0 & \ldots & 0 & 0 \\
(*) & (*) & (*) & (*) & -\bar{g}_{11} W_{11} & \ldots & 0 & 0 \\
(*) & (*) & (*) & (*) & (*) & \ddots & 0 & 0 \\
(*) & (*) & (*) & (*) & (*) & (*) & -\bar{g}_{m m} W_{m m} & 0 \\
(*) & (*) & (*) & (*) & (*) & (*) & (*) & -\tau I_{m}
\end{array}\right]<0
$$

where $(2,2)^{*}=(2,2)+\tau(H \Phi)^{T} \beta^{2} H \Phi$. Dividing (33) by $\tau$, redefining $\left[P_{1}, N_{2}, N_{3}, R, S, W\right]=$ $\tau^{-1}\left[P_{1}, N_{2}, N_{3}, R, S, W\right]$, applying Schur complement to the quadratic term $(H \Phi)^{T} \beta^{2}(H \Phi)$ and defining $\gamma=\beta^{-2}$ leads to

$$
\left[\begin{array}{ccccccccc}
(1,1) & (1,2) & h N_{2}^{T} A_{2} & h N_{2}^{T} A_{3} & \bar{g}_{11} N_{2}^{T} A_{4_{11}} & \ldots & \bar{g}_{m m} N_{2}^{T} A_{4_{m m}} & N_{2}^{T} \Gamma & 0 \\
(*) & (2,2) & h N_{3}^{T} A_{2} & h N_{3}^{T} A_{3} & \bar{g}_{11} N_{3}^{T} A_{4_{11}} & \ldots & \bar{g}_{m m} N_{3}^{T} A_{4_{m m}} & N_{3}^{T} \Gamma & (H \Phi)^{T} \\
(*) & (*) & -h R & 0 & 0 & \ldots & 0 & 0 & 0 \\
(*) & (*) & (*) & -h S & 0 & \ldots & 0 & 0 & 0 \\
(*) & (*) & (*) & (*) & -\bar{g}_{11} W_{11} & \ldots & 0 & 0 & 0 \\
(*) & (*) & (*) & (*) & (*) & \ddots & 0 & 0 & 0 \\
(*) & (*) & (*) & (*) & (*) & (*) & -\bar{g}_{m m} W_{m m} & 0 & 0 \\
(*) & (*) & (*) & (*) & (*) & (*) & (*) & -I_{m} & 0 \\
(*) & (*) & (*) & (*) & (*) & (*) & (*) & (*) & -\gamma I_{m}
\end{array}\right]<0
$$

The LMI (34), which ensures asymptotic stability, is convex in $h$ : if it holds for $\bar{h}>0$ then it is also feasible for all $0 \leq h \leq \bar{h}$; and thus the theorem follows.

\section{B. PROOF OF THEOREM 2}

Let us define $Y_{2}=N_{2}^{-1}, \quad\left[Y_{1}, Y_{R}, Y_{S}, Y_{W_{i j}}\right]=Y_{2}^{T}\left[P_{1}, R, S, W_{i j}\right] Y_{2}$, and assume that $N_{3}=\epsilon N_{2}$, with a tuning scalar $\epsilon$ [22]. Multiplying (34) by diag $\left\{Y_{2}^{T}, \ldots, Y_{2}^{T}, I_{m}, I_{m}\right\}$ and its transpose from

\begin{tabular}{|c|c|c|c|c|c|c|c|c|}
\hline$(1,1)$ & $(1,2)$ & $h A_{2} Y_{2}$ & $h A_{3} Y_{2}$ & $\bar{g}_{11} A_{411} Y_{2}$ & $\ldots$ & $\bar{g}_{m m} A_{4_{m m}} Y_{2}$ & $\Gamma$ & 0 \\
\hline$(*)$ & $(2,2)$ & $\epsilon h A_{2} Y_{2}$ & $\epsilon h A_{3} Y_{2}$ & $\epsilon \bar{g}_{11} A_{4_{11}} Y_{2}$ & $\ldots$ & $\epsilon \bar{g}_{m m} A_{4_{m m}} Y_{2}$ & $\epsilon \Gamma$ & $Y_{2}^{T}(H \Phi)^{T}$ \\
\hline$(*)$ & $(*)$ & $-h Y_{R}$ & 0 & 0 & $\ldots$ & 0 & 0 & 0 \\
\hline$(*)$ & $(*)$ & $(*)$ & $-h Y_{S}$ & 0 & $\ldots$ & 0 & 0 & 0 \\
\hline$(*)$ & $(*)$ & $(*)$ & $(*)$ & $-\bar{g}_{11} Y_{W_{11}}$ & $\ldots$ & 0 & 0 & 0 \\
\hline$(*)$ & $(*)$ & $(*)$ & $(*)$ & $(*)$ & $\ddots$ & 0 & 0 & 0 \\
\hline$(*)$ & $(*)$ & $(*)$ & $(*)$ & $(*)$ & $(*)$ & $-\bar{g}_{m m} Y_{W_{m m}}$ & 0 & 0 \\
\hline$(*)$ & $(*)$ & $(*)$ & $(*)$ & $(*)$ & $(*)$ & $(*)$ & $-I_{m}$ & 0 \\
\hline$(*)$ & $(*)$ & $(*)$ & $(*)$ & $(*)$ & $(*)$ & $(*)$ & $(*)$ & $-\gamma I_{m}$ \\
\hline
\end{tabular}
the left and right, respectively, results in

with $\quad(1,1)=A_{1} Y_{2}+Y_{2}^{T} A_{1}^{T}+h Y_{R}+\sum_{i=1}^{m} \sum_{j=1}^{m} \bar{g}_{i j} Y_{W_{i j}}, \quad(1,2)=Y_{1}-Y_{2}+\epsilon Y_{2}^{T} A_{1}^{T} \quad$ and $(2,2)=-\epsilon\left(Y_{2}^{T}-Y_{2}\right)+h S$. Now, the following decomposition is taken

$$
A_{1}=A_{10}+B_{10} \tilde{K} \quad A_{2}=\tilde{K}^{T} B_{20} \tilde{K} \quad A_{3}=B_{30} \tilde{K}
$$

where $\tilde{K}=\operatorname{diag}\{K, \Omega, \Omega\}$ is an augmented gain matrix and the other matrices involved are defined in (25). Plugging (36) into (35), and defining $\tilde{K} Y_{2}=X$, the LMI (24) follows. Finally, as suggested in [45], the conditions $X^{T} X<\kappa_{x} I$ and $Y^{-1}<\kappa_{y} I$, which are equivalent to

$$
\left[\begin{array}{cc}
-\kappa_{x} I & X^{T} \\
(*) & -I
\end{array}\right]<0 \quad \text { and } \quad\left[\begin{array}{cc}
Y_{2} & I \\
(*) & \kappa_{y} I
\end{array}\right]>0
$$


respectively, would ensure that $\|\tilde{K}\|^{2}=Y_{2}^{-1} X^{T} X Y_{2}^{-1}<\kappa_{x} \kappa_{y}^{2}$. The theorem follows by defining the submatrices $Y_{2}^{(j)}, X^{(j)}$ and imposing conditions like (37) to every submatrix.

\section{REFERENCES}

1. Sipahi R, Niculescu SI, Abdallah CT, Michiels W, Gu K. Stability and stabilization of systems with time delay. Control Systems, IEEE 2011; 31(1):38-65.

2. Smith OJ. A controller to overcome dead time. ISA Journal 1959; 6(2):28-33.

3. Normey-Rico J, Bordons C, Camacho E. Improving the robustness of dead-time compensating PI controllers. Control Engineering Practice 1997; 5(6):801-810.

4. Michiels W, Niculescu S. On the delay sensitivity of smith predictors. International Journal of Systems Science 34 (2003) 543-552; .

5. Zhong QC. Robust control of time-delay systems. Springer Science \& Business Media, 2006.

6. Normey-Rico JE, Camacho EF. Dead-time compensators: A survey. Control Engineering Practice 2008; 16(4):407428.

7. Guzman J, Garcia P, Hägglund T, Dormido S, Albertos P, Berenguel M. Interactive tool for analysis of time-delay systems with dead-time compensators. Control Engineering Practice 2008; 16(7):824-835.

8. Manitius A, Olbrot AW. Finite spectrum assignment problem for systems with delays. Automatic Control, IEEE Transactions on $1979 ; \mathbf{2 4}(4): 541-552$.

9. Artstein Z. Linear systems with delayed controls: A reduction. Automatic Control, IEEE Transactions on 1982; 27(4):869-879.

10. Moon YS, Park P, Kwon WH. Robust stabilization of uncertain input-delayed systems using reduction method. Automatica 2001; 37(2):307-312.

11. Yue D. Robust stabilization of uncertain systems with unknown input delay. Automatica 2004; 40(2):331-336

12. Yue D, Han QL. Delayed feedback control of uncertain systems with time-varying input delay. Automatica 2005; 41(2):233-240.

13. Lozano R, Castillo P, Garcia P, Dzul A. Robust prediction-based control for unstable delay systems: Application to the yaw control of a mini-helicopter. Automatica 2004; 40(4):603-612.

14. Gonzalez A, Garcia P, Albertos P, Castillo P, Lozano R. Robustness of a discrete-time predictor-based controller for time-varying measurement delay. Control Engineering Practice 2012; 20(2):102-110.

15. Karafyllis I, Krstic M. Robust predictor feedback for discrete-time systems with input delays. International Journal of Control 2013; 86(9):1652-1663.

16. Krstic M. Input delay compensation for forward complete and strict-feedforward nonlinear systems. Automatic Control, IEEE Transactions on 2010; 55(2):287-303.

17. Bekiaris-Liberis N, Krstic M. Compensation of time-varying input and state delays for nonlinear systems. Journal of Dynamic Systems, Measurement, and Control 2012; 134(1):011 009.

18. Choi JY, Krstic M. Compensation of time-varying input delay for discrete-time nonlinear systems. International Journal of Robust and Nonlinear Control 2015; 26(4):1755-1776.

19. Karafyllis I, Malisoff M, Mazenc F, Pepe P. Stabilization of nonlinear delay systems: A tutorial on recent results. Recent Results on Nonlinear Delay Control Systems. Springer, 2016; 1-41.

20. Cacace F, Conte F, Germani A, Pepe P. Stabilization of strict-feedback nonlinear systems with input delay using closed-loop predictors. International Journal of Robust and Nonlinear Control 2016; .

21. Fridman E, Shaked U. An improved stabilization method for linear time-delay systems. Automatic Control, IEEE Transactions on 2002; 47(11):1931-1937.

22. Fridman E, Shaked U. A descriptor system approach to $H_{\infty}$ control of linear time-delay systems. Automatic Control, IEEE Transactions on 2002; 47(2):253-270.

23. Chen WH, Zheng WX. On improved robust stabilization of uncertain systems with unknown input delay. Automatica 2006; 42(6):1067-1072.

24. Boukas EK, Liu ZK. Deterministic and stochastic time-delay systems. Springer Science \& Business Media, 2012.

25. Krstic M. Lyapunov tools for predictor feedbacks for delay systems: Inverse optimality and robustness to delay mismatch. Automatica 2008; 44(11):2930-2935

26. Léchappé V, Moulay E, Plestan F, Glumineau A, Chriette A. New predictive scheme for the control of LTI systems with input delay and unknown disturbances. Automatica 2015; 52:179-184.

27. Roh YH, Oh JH. Robust stabilization of uncertain input-delay systems by sliding mode control with delay compensation. Automatica 1999; 35(11):1861-1865.

28. Bresch-Pietri D, Krstic M. Adaptive trajectory tracking despite unknown input delay and plant parameters. Automatica 2009; 45(9):2074-2081.

29. Kamalapurkar R, Fischer N, Obuz S, Dixon WE. Time-varying input and state delay compensation for uncertain nonlinear systems. IEEE Transactions on Automatic Control 2015; 61(3):834-839.

30. Chen WH, Ohnishi K, Guo L. Advances in disturbance/uncertainty estimation and attenuation. Industrial Electronics, IEEE Transactions on 2015; 62(9):5758-5762.

31. Chen WH, Yang J, Guo L, Li S. Disturbance observer-based control and related methods: An overview. Industrial Electronics, IEEE Transactions on 2016; 63(2):1083-1095.

32. Sariyildiz E, Ohnishi K. Design constraints of disturbance observer in the presence of time delay. Mechatronics (ICM), 2013 IEEE International Conference on, IEEE, 2013; 69-74.

33. Wang QG, Hang CC, Yang XP. Single-loop controller design via IMC principles. Automatica 2001; 37(12):20412048 . 
34. Zheng Q, Gao Z. Predictive active disturbance rejection control for processes with time delay. ISA transactions 2014; 53(4):873-881.

35. Chen M, Chen WH. Disturbance-observer-based robust control for time delay uncertain systems. International Journal of Control, Automation and Systems 2010; 8(2):445-453.

36. Guo L, Chen WH. Disturbance attenuation and rejection for systems with nonlinearity via DOBC approach. International Journal of Robust and Nonlinear Control 2005; 15(3):109-125.

37. Isidori A. Nonlinear control systems. Springer Science \& Business Media, 2013.

38. Zhong QC, Rees D. Control of uncertain LTI systems based on an uncertainty and disturbance estimator. Journal of dynamic systems, measurement, and control 2004; 126(4):905-910.

39. He Y, Wu M, She JH. Improved bounded-real-lemma representation and $H_{\infty}$ control of systems with polytopic uncertainties. Circuits and Systems II: Express Briefs, IEEE Transactions on 2005; 52(7):380-383.

40. Cao YY, Lam J, Sun YX. Static output feedback stabilization: an ilmi approach. Automatica 1998; 34(12):16411645.

41. Marler RT, Arora JS. The weighted sum method for multi-objective optimization: new insights. Structural and multidisciplinary optimization 2010; 41(6):853-862.

42. Fridman E. Introduction to time-delay systems: Analysis and control. Springer, 2014.

43. Solomon O, Fridman E. New stability conditions for systems with distributed delays. Automatica 2013; 49(11):3467-3475.

44. Li H, Fu M. A linear matrix inequality approach to robust h filtering. IEEE Transactions on Signal Processing 1997; 45(9):2338-2350.

45. Siljak D, Stipanovic D. Robust stabilization of nonlinear systems: the LMI approach. Mathematical problems in Engineering 2000; 6(5):461-493. 